\title{
Norm Emergency through Argumentation
}

\author{
S. Heras, N. Criado, E. Argente and V. Julián
}

\begin{abstract}
Open societies are situated in dynamic environments and are formed by heterogeneous autonomous agents. In order to ensure social order, norms have been employed as coordination mechanisms. However, the dynamical features of open systems may cause that norms loose their validity and need to be adapted. Therefore, this paper proposes a new dialogue game protocol for modelling the interactions produced between agents that must reach an agreement on the use of norms. An application example has been presented for showing both the performance of the protocol and its usefulness as a mechanism for managing the solving process of a coordination problem through norms.
\end{abstract}

Index Terms-Norm Emergency, Dialogue Games, Normative MAS

\section{Motivation}

$\mathbf{N}$ OWADAYS, Multi-agent Systems (MAS) research on addressing the challenges related to the development of open distributed systems is receiving an increasing interest [8]. The main features of open systems are: (i) they are populated by heterogeneous agents which can enter or leave the system dynamically; and (ii) they are situated in dynamic environments. Therefore, their entities might be unknown and none assumption about their performance can be done [2]. As a consequence, mechanisms for coordinating their behaviours and ensuring social order are essential. In this sense, social factors are becoming more and more important to coordinate interactions in dynamic open worlds. Works on Normative Theory have been applied into the MAS area as a mechanism for facing up with undesirable and unexpected behaviours [7]. These regulatory mechanisms attempt to guarantee a globally efficient coordination in open systems taking into account the impossibility of controlling (the majority of) the agents and services directly.

Two different approximations have been considered as alternatives to the definition of norms: (i) off-line design, where the system designer defines the normative system statically [15]; and (ii) automatic emergence, which analyses how norms can emerge inside a group of agents [16]. The latter is more suitable for open systems, in which structural, functional and environmental changes might occur [17]. Therefore, dynamical situations may cause that the norms that regulate an organization lose their validity or should be adapted. In this second case, techniques for reaching an agreement among agents on the employment of norms are needed.

This research is aimed at providing a mechanism for managing norm emergence in open environments. THOMAS [4], [5], a development architecture for Virtual Organisations (VO) [3], has been selected as a suitable environment to test this proposal. The main idea that inspired this architecture was

S. Heras, N. Criado, E. Argente and V. Julián are with Universidad Politécnica de Valencia.

E-mail: sheras, ncriado, eargente, vinglada@dsic.upv.es to give support a better integration between the standardised service-oriented computing technologies and the MAS paradigm. Both technologies can complement the strengths of each other: (i) service standards provide an infrastructure for the interaction among agents; (ii) MAS offer a more general and complex notion of Service Oriented Architectures (SOA); and (iii) intelligent and social capabilities of agents allow defining complex services. In THOMAS, the coordination among heterogeneous agents is achieved by means of norms. Thus, a normative language for formalising constraints on agent behaviours has been developed [1].

Our approach is to apply dialogue games as an argumentation technique to model the interaction among agents that must reach an agreement about the normative context. Dialogue games are interactions between players where each one moves by advancing locutions in accordance to some pre-defined set of rules [11]. In MAS, they have been used to specify communication protocols [10] and to evaluate reputation [12]. However, the application of argumentation techniques to the definition of norms is a novel area of research.

This paper is structured as follows. Section 2 briefly introduces background of this work; the THOMAS architecture and the dialogue game protocol on which the work is based. Section 3 describes the dialogue game protocol specification. Section 4 shows an example of the protocol applied to solve a coordination problem in THOMAS. Finally, section 5 summarises the main conclusions drawn from this research.

\section{BACKGROUND}

This section briefly introduces the THOMAS architecture and the dialogue game adapted to cope with the objective of our research.

\section{A. THOMAS Architecture}

THOMAS architecture [4], [5] has been proposed for the generation of Virtual Organisations (VO) in open environments. The main idea that inspired THOMAS architecture was to achieve a better integration between the standardised service-oriented computing technologies and the MAS paradigm. Therefore, every operation that can be performed in the architecture (even the THOMAS management functionalities) is described and offered by means of Web Services standards. A description of the THOMAS architecture can be found at ${ }^{1}$.

The THOMAS architecture feeds on the FIPA ${ }^{2}$ architecture, but extends its main components - the Agent Management System (AMS) and the Directory Facilitator (DF) — into

\footnotetext{
${ }^{1}$ http://www.fipa.org/docs/THOMASarchitecture.pdf

${ }^{2}$ http://www.fipa.org
} 


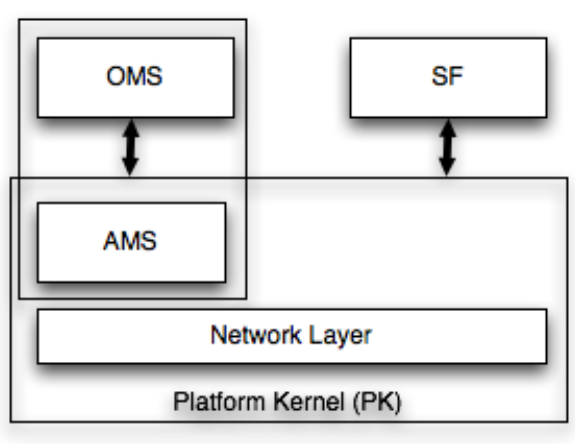

Fig. 1. Thomas architecture

an Organization Management System (OMS) and a Service Facilitator (SF), respectively. More specifically, the main components of THOMAS architecture are (see Figure 1):

- Platform Kernel (PK), that deals with basic agent management services and it can be provided by any FIPAcompliant platform. Its functionality is related with the agent life-cycle and the network communication layer.

- Service Facilitator (SF), which is a service manager that registers services provided by external entities and facilitates service discovering for potential clients. The SF can be considered as a yellow pages server.

- Organization Management System (OMS), which is responsible of the management of virtual organizations, taking control of their underlying structure, the roles played by the agents inside the organization and the norms that rule the system behaviour.

Open MAS require mechanisms for controlling agents behaviours and ensuring social order. The THOMAS architecture proposes the use of a normative system to cope with this requirement. Thus, the THOMAS architecture allows defining norms that prescribe agent rights and duties in terms of who can provide a service, when and under which circumstances. With this aim, a normative language for formalising constraints on agent behaviours has been developed [1]. This language allows the specification of norms that define deontic prescriptions to control access to services in THOMAS.

However, as a consequence of the dynamicity of the environments that THOMAS is intended for developing and the heterogeneity of their agents, changes in the normative context frequently occur. Thus, a mechanism for adapting the normative context to the current state of the VO is desirable. This adaptation process can be formalised as a general coordination problem. In [9], coordination is defined as 'managing dependencies between activities'. Therefore, a coordination problem arises when a group of agents share a goal which fulfilment requires the cooperation among the activities performed by agents [14]. In this research, the problem to cope with is to reach an agreement about the definition of a normative context. The interaction among the agents that participate in the agreement process is coordinated by means of a dialogue game protocol.

\section{B. Argument from Expert Opinion}

We have adapted a general dialogue game, the Argumentation Scheme Dialogue (ASD) [13] to formalise the interaction among agents that argue about a normative context. Our work is based on this general dialogue game, which extends traditional dialogue games with certain stereotyped patterns of common reasoning called argumentation schemes. Concretely, we have instantiated this game by making use of a specific argumentation scheme, the Argument from Expert Opinion [18] that captures the way in which people evaluates the opinions (recommendations about norms in our context) of experts (agents with some knowledge about a set of norms to recommend). The structure of the scheme is the following:

- Major Premise: Source E is an expert in field F containing proposition A.

- Minor Premise: E asserts that proposition A (in field F) is true (false).

- Conclusion: A may plausibly be taken to be true (false). Moreover, this scheme has also a set of critical questions, which represent the possible attacks that can be made to rebut the conclusion drawn from the scheme and hence, are very useful for coordinating the dialogue:

1) Expertise: How credible is $E$ as an expert source?

2) Field: Is $E$ an expert in the field $F$ that $A$ is in?

3) Opinion: What did E assert that implies A?

4) Trustworthiness: Is E personally reliable as a source?

5) Consistency: Is A consistent with what other experts assert?

6) Backup Evidence: Is E's assertion based on evidence?

\section{Dialogue Game Protocol Specification}

In this section, we explain the main features of the dialogue game protocol proposed. First, the social structure that allows agents to evaluate arguments of other agents is shown. Based on it, the dialogue game protocol is specified. Finally, how agents can pose arguments and rebut attacks is also described.

\section{A. Social Structure}

Following the normative emergence approach, in this paper we propose the use of a service of normative assistance to solve coordination problems in VOs. This service is provided by a set of Normative Assistant (NA) agents that recommend the appropriate modifications to fit the operation of a VO to the current situation by adapting its normative context. The normative assistance services are published by the THOMAS SF and thus, they can be publicly requested by all agents of the organization.

Therefore, whenever an agent of the system wants to solve a coordination problem, it assumes the role of initiator and requests the $\mathrm{SF}$ a list of providers of the normative assistance service. Then, among these providers, it may select a subset of NAs with agents that it personally considers as 'friends' (previously known agents, if any). This friendship relation comes from past recommendation processes where the initiator was involved. The experience-based friendship relations of all agents of the system can be represented by a social network 
abstraction. The network topology would be implicitly defined through the confidence relations that an agent has with its friends and is, thus, decentralised. In this network, nodes would represent agents and links would be friendship relations between them (labelled with confidence values).

Therefore, since the first recommendation dialogue where an agent was engaged in as initiator, this agent keeps a list of all the agents that participated in the dialogue and its final assessment about the confidence about the recommendations received from other agents. This confidence degree $c_{i j} \in$ $[-1,1]$ is updated at the end of each recommendation process by using a discrete value $u_{j} \in\{-1:$ inappropriate, 0 : useless or $1:$ useful $\}$ that stands for the final usefulness of each recommendation received by the initiator. In addition, the initiator also informs the NAs that participated in the dialogue of the usefulness value of their recommendations. With this value, each NA can update its expertise degree $e_{j}$ as norms recommender. These degrees are computed by using equations 1 and 2:

$$
\begin{gathered}
c_{i j}=\frac{\sum_{k=1}^{K} u_{j(k)}}{K} \\
e_{j}=\frac{\sum_{i=1}^{I} c_{i j}}{\operatorname{deg}^{+}\left(a_{j}\right)}
\end{gathered}
$$

In the equations, $u_{j(k)}$ is the usefulness degree of the recommendation $k$ that an agent accepted from its friend $a_{j}$, $K$ is the total number of recommendations accepted by an agent and $\operatorname{deg}^{+}\left(a_{j}\right)$ is the centrality indegree of $a_{j}$ (the number of agents that consider this agent as friend). Note that, although confidence and expertise degrees could be considered subjective and could be risky for an agent to believe them by default, these measures are defeasible and the interaction protocol explained in the next section allows agents to argue about them.

We also assume that each NA agent stores the set of norms that were effective to solve a coordination problem in the VOs where it has participated in a norm database. In addition, for every set of norms, it also stores the attributes that characterised the type of problem addressed. Therefore, the knowledge about the solving process of the different coordination problems is distributed across the network and each NA agent only has a partial view based on its own experience. Moreover, if a NA is asked for a set of norms to deal with a coordination problem that it has never been faced with, the agent can also propagate the query to its neighbours in the network by using its friendship relationships (i.e. such NA agents that have eventually provided this NA agent with recommendations in the past). When all NAs have made their proposals, the initiator has been presented with several recommendations about sets of norms. Then, it selects the best proposal by using the proposed dialogue game protocol.

Finally, note that each agent is assumed to have its own reasoning mechanism for evaluating preferences, matching them with its norm database and proposing recommendations. In addition, agents must also know a set of inference rules and the scheme from expert opinion to be able to create arguments. The definition of the individual reasoning mechanisms of agents (e.g. how they manage arguments and evaluate the usefulness degree of recommendations) is out of the scope of this paper. We have mainly focused here on formalising the dialogue-based interaction protocol. Following, the specification of this interaction protocol is detailed.

\section{B. Protocol Specification}

The dialogue game protocol proposed in this paper is an application of the ASD game instantiated with the Argument from Expert Opinion scheme presented in [6]. On one hand, the protocol assumes the existence of:

- A finite set of players denoted Agents, with elements $\left\{A g_{i}, N A_{1}, \ldots, N A_{n}\right\}$, consisting of the agent $A g_{i}$ that plays the Initiator role and starts the dialogue and the set of normative assistants $\left\{N A_{1}, \ldots, N A_{n}\right\}$ in its social network that play the role of recommendations proponents.

- A finite set of recommended items, denoted Items, with elements $\left\{i_{1}, \ldots, i_{m}\right\}$. Each item consists of the pair $i=<I D$, type $>$, where $I D$ is an unique identifier for each item and type represents the class of the item (e.g. normative set).

- A finite set of discrete values that represent the feedback that an agent offers to the dialogue participants about their recommendation, denoted Usefulness, with elements -1: Inappropriate, 0: Useless or 1: Useful.

- A finite set of variables that represent the agent's preferences when it asks for a recommendation, denoted Preferences, with elements $P=\left\{p_{1}, \ldots, p_{q}\right\}$.

- A function preference: $P \rightarrow 2^{F \times V}$, which maps each Preference of an agent to a set of pairs $\langle f, v\rangle$, where $\mathrm{f} \in$ Features and $\mathrm{v} \in$ Values.

- A function feedback: Item $\rightarrow$ Usefulness, which maps an Item that an agent has recommended to one of the possible values of usefulness.

Finally, the interaction protocol between the agents of the network has been modelled as a formal dialogue game with the components identified by McBurney and Parsons [11]:

- Commencement Rules: The process consists in a set of parallel dialogues between the initiator and the NAs (who do not speak directly between them). In each step of the dialogue, either the initiator makes a move or the NA answers it by posing the permissible locutions following the dialogue rules. Note that, although the information provided by a NA is not directly accessible by another, the initiator acts as a mediator and is able to use this information when speaking with other NAs. The dialogue starts when an agent asks the THOMAS SF component for a set of NAs. Then, the initiator of the dialogue uses its list of friends to select a subset of those NAs and send them a request for a normative set recommendation. When the NAs have received the recommendation request with the preferences of the initiator, they use their own reasoning mechanisms to match these preferences with their knowledge database and offer a recommendation. 
Eventually, NAs can decide not to engage in the recommendation dialogue.

- Locutions:

- Statements: they are the permissible locutions in the dialogue and their compounds (propose(Item, Preferences), accept, reject, noCommitment(Item) and assert(Data)).

- Questions: propose(Item,Preferences)? is used to request recommendations from friends. Also, the locution assert $(\{$ Preferences $\})$ ? asks the initiator for more information about its preferences when there are some unspecified. Agents are not committed to answering questions.

- Critical Attacks (CA): the locution pose(CA) poses a critical attack associated with one of the critical questions of the Argument from Expert Opinion scheme. In our model we assume (a) that all NAs have some knowledge about the items of the domain and hence, every NA can be considered expert to some extent (Field question); (b) that NAs are rational and always propose the recommendation that, using their reasoning mechanisms, better fits the preferences of the initiator (Opinion question) and (c) that NAs are honest and their recommendations and arguments are based on their own knowledge (Backup evidence question). Thus, permissible attacks to recommendations are: (i) questioning the degree of expertise of the proponent (Expertise question); (ii) demonstrating that the proponent is not personally reliable as recommender (Trustworthiness question); or (iii) demonstrating that the proponent's recommendation is not consistent with the one of other expert with equal or greater degree of expertise (Consistency question). The burden of proof in the case of the Trustworthiness and Consistency attacks falls on the initiator and therefore, if the attack is challenged, it is committed to providing the proponent with arguments that justify those criticisms.

- Challenges: why(locution)?, where locution can be a recommendation proposal or a critical attack, requests arguments that support them.

- Commitment Rules:

- Before the assertion of the locution propose(Item, Preferences), if proponents have different recommendations that match the preferences of the initiator, they are committed to ask it for specifying those preferences that could stand out a recommendation from the rest.

- The assertion of the locution propose(Item, Preferences) commits the proponent to the assumptions that the game makes about the critical questions of the Argument from Expert Opinion scheme.

- The assertion of the locution noCommitment(Item), withdraws the recommendation made by the proponent and frees it from all commitments.

- The assertion of the locution why(propose(Item, Preferences))? commits the proponent of the recom- mendation either to providing the initiator with a justification about its proposal or to withdrawing it.

- If a critical attack pose $(C A)$ is challenged, the initiator is committed to providing arguments to justify it.

- Dialogue Rules:

1) The initiator's request opens the dialogue and each NA can answer it by a request for more information about the properties that characterise the problem, a normative set proposal or a rejection to provide the assistance service.

2) NAs can ask for more information while the initiator accedes to provide it. Finally, the NA makes a proposal (proponent role) or rejects to provide the service.

3) Each normative set proposal can be followed by a request for an argument supporting the recommendation, or by its acceptance or rejection.

4) If a recommendation is challenged, the proponent must show its argument for recommending such normative set or withdraw the recommendation.

5) The initiator can reply to the argument of a NA by accepting the recommendation, by rejecting it or by posing a critical attack associated with the Argument from Expert Opinion explained in section II-B. Possible attacks in our context are: questioning the degree of expertise of the NA or demonstrating that the recommendation of the NA is not consistent with other NA recommendations with equal or greater degree of confidence or expertise.

6) Trustworthiness and Consistency attacks can also be challenged by the NA. Then, the initiator must provide an argument supporting the attack.

7) NAs can rebut attacks or else, withdraw their recommendations.

8) Finally, the initiator can accept the argument of a NA and choose its recommendation, preliminary accept its argument but pose another attack or reject the argument and hence, the recommendation (ending the dialogue with this NA).

- Termination Rules: The initiator agent keeps at every moment the entire control of the recommendation process. The preferences of the initiator do not change during the dialogue and proponents are assumed to be rational and honest. Thus, once a recommendation has been proposed, it cannot be changed. Proponents are only allowed to propose items, to answer challenges and critical attacks and to withdraw their recommendations. The game ends when the initiator has taken a decision among the set of available recommendations, which can happen at any time during the dialogue. Afterwards, the proponents are informed and receive the accept/reject locutions to their recommendations. However, after a maximum time is exceeded, the recommendation partially accepted to this step of the dialogue is taken as the final decision. 


\section{Decision-Making Process of NAs}

The basic decision policy that follows every NA is to do its best to convince the initiator that its normative set recommendation is the most appropriate one to solve the coordination problem. There are two different types of arguments that NAs can use to persuade the initiator: arguments for justifying a recommendation and arguments for rebutting an attack. An argument of the former type consists in a set of common attributes among the problem characterisation and the proposed solution. Regarding the rebutting arguments, they consists on a partial ordering relation between confidence or expertise degrees. The simplest case is to rebut an expertise attack, since the NA can only show its expertise degree (note that expertise degrees are private and this attack is thought to provide the initiator with this information).

In the case of a trustworthiness attack, the initiator $i$ attacks the recommendation of the proponent $p$ because it has received a different recommendation from other NA $n$ with a higher confidence degree for the initiator. Then, $p$ can rebut the attack if the conditions expressed on equation 3 hold:

$$
\begin{gathered}
\text { Argument } A R=(c,<, c) \\
\text { partial ordering relation } \delta=\{<\} \\
\text { attacks } \subseteq \text { ARe }(a) \times A R \\
\\
\text { Case }(b) \quad\left(c_{n p},<, c_{i n}\right) \text { attacks }\left(c_{i p},<, c_{\text {in }}\right) \text { iff } \\
c_{p n}<c_{i p} \\
\left(c_{i n},<, c_{p k}\right) \text { attacks }\left(c_{i p},<, c_{i n}\right) \text { iff } \\
0<c_{i p}<c_{i n}<c_{p k}
\end{gathered}
$$

In case (a), $p$ can rebut the attack if it personally knows $n$ and its confidence degree in this NA $c_{p n}$ is lower than the confidence degree $c_{i p}$ of the initiator in $p$. In case (b), $p$ can rebut the attack if the recommendation was propagated to the NA $k$ and the confidence degree $c_{p k}$ of $p$ in this NA is higher than the confidence degree $c_{i p}$ that the initiator has in $p$ and also the confidence degree $c_{i n}$ that the initiator has in the NA $n$.

In the case of a consistency attack, the initiator $i$ attacks the recommendation of the proponent $p$ because it has received a different recommendation from other NA $n$ with a higher expertise degree for the initiator. Then, $p$ can rebut the attack if the conditions expressed on equation 4 hold:

$$
\begin{gathered}
\text { Argument } A R=(e,<, e) \\
\text { partial ordering relation } \delta=\{<\} \\
\text { attacks } \subseteq A R \times A R \\
\left(e_{n},<, e_{k}\right) \text { attacks }\left(e_{p},<, e_{n}\right) \text { iff } \\
e_{p}<e_{n}<e_{k}
\end{gathered}
$$

In this case, $p$ can rebut the attack if the recommendation was propagated to the NA $k$ and the expertise degree $e_{k}$ of this NA $k$ is higher than the expertise degree $e_{p}$ of $p$ and also the expertise degree $e_{n}$ of the NA $n$.

\section{Normative Context Definition}

In order to show the operation of our dialogue game protocol, we have applied it for solving a coordination problem.
More concretely, the addressed problem consists in selecting the most suitable norms for a new organization that an agent wants to create.

\section{A. Problem Formalization}

In our approach, coordination is achieved by means of norms that regulate the activities of agents. Therefore, a coordination problem is formalised as a structure of the type $\gamma$ where $\gamma=\left\{\gamma_{0}, \gamma_{1}, \ldots, \gamma_{k}\right\}$ is a set of attributes or properties that characterise the problem; $\gamma_{i} \in D_{i}$, being $D_{i}$ the domain associated to the property $i$.

In the application example, the addressed problem consists on the definition of the normative context for a new VO according to its desirable features. This problem is a particular case of a coordination problem. Thus, a problem of normative context definition is defined as $\gamma$ where $\gamma=\{s, m, v, c, f\}$ and

- $s=<w h o, h o w>$ is a property that determines who can change the structural components of the system (i.e. roles, units and norms); where who $\in\{$ none, supervisor, all $\}$ and how $\in\{$ increase, decrease, modify $\}$.

- $m \in \mathbb{N}$ is the property that specifies the cardinality of the $\mathrm{VO}$ members.

- $v \in\{$ none, supervisor, members, all $\}$ is the visibility property that establishes the access rights to the information of the VO.

- $c \in\{$ none, supervisor, all $\}$ is the property that determines if the expulsion service can be used as a control mechanism.

- $f=<$ who, how $>$ is the property that defines who and how the functionalities, i.e. services, of the VO can be changed; where who $\in\{$ none, supervisor, all $\}$ and how $\in\{$ increase, decrease, modify $\}$.

Thus, items in this domain correspond to normative sets (i.e. $<I D$, normativeSet $>$ ) and the preferences of the initiator correspond to the properties that characterise the coordination problem (i.e. $\gamma$ ).

\section{B. Application Example}

The social network of normative assistants can be implemented in THOMAS as a VO (named NormativeAssistance). It is a group of agents formed by all agents that can provide normative assistance (Assistants) to other agents. Therefore, agents in charge of the maintenance of the normative context that regulates a specific group can request an advice for norms effective in some scenario. All the communication and functionalities specified by the protocol are carried out as services.

As an example, Figure 2 contains an overview of the services performed by a set of agents which are playing the proposed dialogue game. Let us consider the case that an agent (initiator) has requested the service for creating a new VO in THOMAS. In this situation, the agent needs to define the normative context that will regulate its VO. However, this agent does not know a priory the suitable set of norms for its VO. Thus, it can ask for a NormativeAssistance service to 


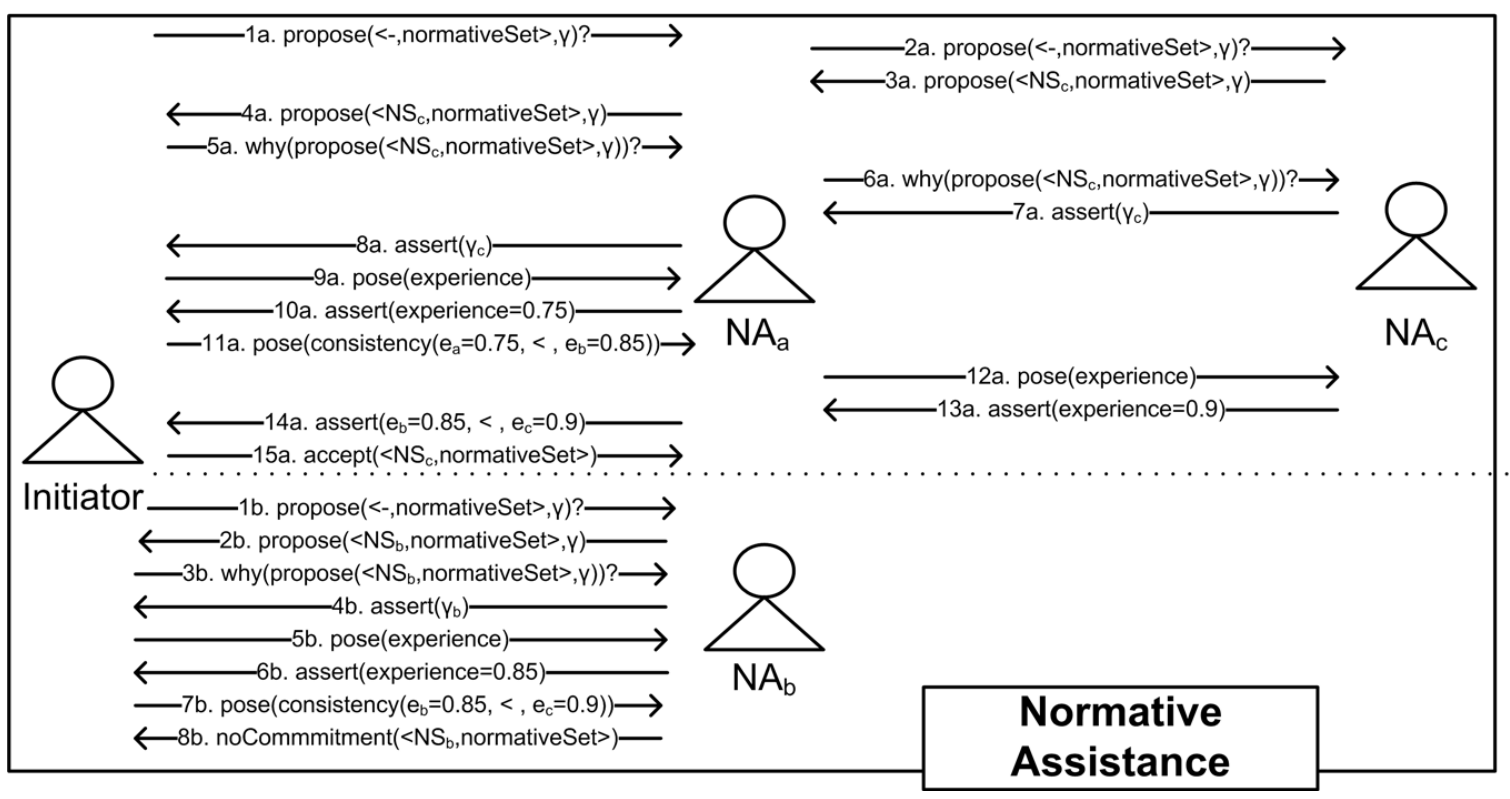

Fig. 2. Example of an execution of the proposed dialogue game

the SF, who will apply its techniques for service searching and composition to discover this recommendation service. If it exists, the initiator agent will obtain the list of service providers from the SF.

As previously mentioned, the initiator selects the NAs from the provider list according to its previous experiences. In this example, the initiator requests the NormativeAssistance service to $N A_{a}$ and $N A_{b}$ (Figure 2 steps $1 \mathrm{a}$ and $1 \mathrm{~b}$ ). This service request implicitly utters the propose locution which starts the dialogue game. Therefore, the initiator carries out two different dialogues concurrently (labelled as $a$ and $b$ ). The $N A_{a}$ does not have the requested information in its norm database and thus, it propagates the request to its friend $N A_{c}$ (step 2a). Thus, the set of players in this example is defined as Agents $=\left\{\right.$ initiator, $\left.N A_{a}, N A_{b}, N A_{c}\right\} . N A_{c}$ queries its norm database and proposes the normative set $N S_{c}$ (step 3a and its propagation in step 4a). Moreover, the $N A_{b}$ proposes $N S_{b}$ as a solution to the coordination problem characterized by $\gamma$ (step 2b). Since the initiator does not have enough information for choosing between $N S_{c}$ and $N S_{b}$, it requests a justification of the recommended normative sets (steps 5a, its propagation $6 \mathrm{a}$ and step $3 \mathrm{~b}$ ). After receiving the justification of the proposed recommendation (steps $7 \mathrm{a}$, its propagation $8 \mathrm{a}$ and step $4 \mathrm{~b}$ ) the initiator still does not have enough information for taking a decision. Thus, it poses an expertise attack (steps 9a and $5 \mathrm{~b}$ ) to $N A_{a}$ and $N A_{b}$, which answer by informing about their expertise degree in steps $10 \mathrm{a}$ and $6 \mathrm{~b}$, respectively. Since the expertise of $N A_{b}$ is higher than $N A_{a}$, the initiator poses a consistency attack to $N A_{a}$ (step 11a). As a consequence, $N A_{a}$ poses an expertise attack to $N A_{c}$ (step 12a) to know its expertise degree (provided in step 13a). Hence, $N A_{a}$ is able to rebut the confidence attack by showing that the expertise level of $N S_{c}$ is higher than $N S_{b}$ (step 14a). Then, the initiator poses a consistency attack to $N A_{b}$ which cannot rebut it and hence, withdraws its recommendation (steps $7 \mathrm{~b}$ and $8 \mathrm{~b}$, respectively).
Finally, the initiator accepts the normative set $N S_{c}$ as the best solution for the current coordination problem (step 15a).

After this recommendation dialogue, the initiator applies the normative set $N S_{c}$ in its $\mathrm{VO}$ and evaluates its effectiveness. The definition of the recommendation evaluation process carried out by agents is out of the scope of this paper. Therefore, the initiator is able to update its confidence degrees of the agents that engaged in the dialogue $\left(N A_{a}, N A_{b}\right.$ and $N A_{c}$ ). This last agent is added to its friend list. In addition, the initiator sends feedback information about the utility of the recommendation to all NAs, which update their expertise degrees.

\section{CONClusion}

This paper addresses the problem consisting in the dynamical definition of norms for agent societies. In open societies, which are situated in a dynamic environment and are formed by heterogeneous autonomous agents, the existence of mechanisms for adapting and modifying norms is essential. Therefore, our approach proposes the employment of dialogue games to model the interactions produced between agents that must reach an agreement on the use of norms. This work takes as basis well known proposals on dialogue games. In addition, the THOMAS architecture has been used as an infrastructure for the dialogues among agents.

We have adapted the ASD dialogue game to fit our objective of supporting norm emergence in an open and social context. Finally, an application example has been presented in order to show both the performance of the dialogue game protocol and its usefulness as a mechanism for managing the solving process of a coordination problem through norms. As future work we plan to apply this approach to more complex scenarios in which a more elaborated process is required for solving these coordination problems. 


\section{ACKNOWLEDGMENT}

This work was partially supported by CONSOLIDERINGENIO 2010 under grant CSD2007-00022, by the Spanish government and GVA funds under TIN2006-14630-C0301 and PROMETEO/2008/051 projects and by the FPU grant AP2007-01256 awarded to N. Criado.

\section{REFERENCES}

[1] Argente, E., Criado, N., Julián, V., Botti, V.: Norms for Agent Service Controlling. EUMAS-08, pp. 1-15, (2008).

[2] Artikis, A., Pitt, J. :A formal model of open agent societies. In Proceedings of the Fifth international Conference on Autonomous Agents (AGENTS). ACM, New York, NY, 192-193, (2001).

[3] Boella, G., Hulstijn, J., van der Torre, L.: Virtual organizations as normative multiagent systems. HICSS IEEE Computer Society, (2005).

[4] Carrascosa, C., Giret, A., Julián, V., Rebollo, M., Argente, E., Botti, V.: Service Oriented Multi-agent Systems: An open architecture. AAMAS09, pp. 1291-1292, (2009).

[5] Giret, A., Julian, V., Rebollo, M., Argente, E., Carrascosa C., Botti,V.:An Open Architecture for Service-Oriented Virtual Organizations. Seventh international Workshop on Programming Multi-Agent Systems(PROMAS), pp. 74-88, (2009).

[6] Heras, S., Navarro, M., Botti ,V., Julián, V.: Applying Dialogue Games to Manage Recommendation in Social Networks. ArgMAS-09, pp. 55-70, (2009).

[7] López y López, F., Luck, M., d'Inverno, M.: Constraining autonomy through norms. AAMAS-02, pp. 674-681, (2002).
[8] Luck, M., McBurney, P., Shehory, O. and Willmott, S.:Agent Technology: Computing as Interaction (A Roadmap for Agent Based Computing). University of Southampton, (2005).

[9] Malone, T., Crowston, K.: The Interdisciplinary Study of Coordination. ACM Computing Surveys, 1994 (March), vol. 26, no. 1, pp. 87-119, (1994).

[10] McBurney, P., Parsons, S.: Dialogue Games in Multi-Agent Systems. Informal Logic. Special Issue on Applications of Argumentation in Computer Science. Vol. 22, no. 3, pp. 257-274, (2002).

[11] McBurney, P., Parsons, S.: Games that Agents Play: A Formal Framework for dialogues between Autonomous Agents. Journal of Logic, Language and Information, vol. 12, no. 2, pp. 315-334, (2002).

[12] O'Donovan, J., Smyth, B.: Trust in recommender systems. 10th International Conference on Intelligent User Interfaces, pp. 167-174, (2005).

[13] Reed, C., Walton. D.: Argumentation Schemes in Dialogue. In Dissensus and the Search for Common Ground, OSSA-07, volume CD-ROM, pp. 1-11, (2007).

[14] Schelling, T.: The Strategy of Conflict. Harvard University Press, Cambridge, (1960).

[15] Shoham, Y., Tennenholtz, M.: On social laws for artificial agent societies: off-line design. Artificial Intelligence, v.73 n.1-2, p.231-252, (1995).

[16] Sen, S., Airiau, S.: Emergence of norms through social learning. In Proceedings of the International Joint Conference on Artificial Intelligence(IJCAI), pp. 1507-1512, (2007).

[17] Verhagen, H.: Norm Autonomous Agents. PhD thesis, Dept. Computer Science, Stockholm University, (2000).

[18] Walton, D.: Appeal to Expert Opinion. Penn State Press, (1997). 